\title{
Active Thermography to analyze the real-time Plants response to UV-B irradiation
}

\author{
by M. Rippa* and P. Mormile*
}

\begin{abstract}
*Institute of Applied Sciences and Intelligent Systems, National Council of Research of Italy (CNR), via Campi
\end{abstract} Flegrei 34, 80078, Pozzuoli (Na), Italy, m.rippa@isasi.cnr.it

\begin{abstract}
The effects that different doses of UV-B radiation can induce on plants have been analyzed in a great number of papers present in the literature but many aspects of the response mechanisms remain to be elucidated. In the present work, to the best of our knowledge, for the first time the UV-B - plants interaction is analyzed by the use of active thermography. The study highlights new aspects of the physiological response of the plants to UV-B radiation and it opens new opportunites for the use of the thermography as an important tool for real-time analysis of plants under UV-B.
\end{abstract}

\section{Introduction}

Ultraviolet Radiation B (UV-B) is a part of the electomagnetic spectrum in the range $280-320 \mathrm{~nm}$ which represent only about $1.5 \%$ of the total solar radiation. Its interaction with the vegetable world is of particular interest because this small part of the solar spectra, according to the UV-B energy dose and the type of plant, induces the accumulation of secondary plant metabolites constituted of antioxidant elements (phenolic compounds, carotenoids, anthocyanins and glucosinolates), highly positive for human health [1]. At same time, also various damaging effects relative to their physiological processes or at DNA level causing heritable mutations [2]. The responses mechanisms of the plants to the UV-B radiation have been analyzed in many works in the last decades $[3,4]$, but most of these mechanism aren't yet clear. In the great majority of these works, plants are irradiated using different protocol and later the effects induced are studied, with a lack of real-time analysis. In this view, the infrared thermography can give an important contribution. This tecnique is a non-destructive method used for inspection and analysis in a great number of research fields. In particular, in literature passive and active thermorgraphy approaches have been used to evaluate important parameters of plant physiology such as transpiration rates, local water content, heat capacity of the leaves and the water flow velocity $[5,6]$. In this work, the UV-B - plant interaction is analyzed by the use of infrared thermography. We use two differents active approaches to analyze the plant response to UV-B radiation: long-pulse thermography using as heat source different doses of UV-B radiation, monitoring the thermal plant response in real-time, and lock-in thermography using a conventional halogen lamp, realized before and after UV-B irradiation, in order to compare the physiologic states of the plant when irradiated with different doses.

\section{Materials and methods}

The thermal response of the leaves during and after UV-B irradiation was monitored in real-time using an infrared camera FLIR X6580 sc with a indium antimonide (InSb) detector (spectral range 1.5-5.4 $\mu$ m, FPA 640x512 pixels and NETD $\sim 20 \mathrm{mk}$ at $25^{\circ} \mathrm{C}$ ) cooled to $75 \mathrm{~K}$ by an integrated stirling cycle. A lens with a $50 \mathrm{~mm}$ focal distance (spectral band 3.5-5 $\mu \mathrm{m}$ and IFOV $0.3 \mathrm{mrad}$ ) was used. Different doses of UV-B radiation $\left(0.4,1.0,1.8,3.6,6.0 \mathrm{~kJ} / \mathrm{m}^{2}\right.$ ) was applied to some selected leaves of a plants belonging to the species Epipremnum aureum, a very common pot plant. Two fluorescent tubes (Philips TL20W/12) were used as UV-B source. The distance between the tubes and the leaf under investigation was fixed. The power radiation was measured using a photo-radiometer (Delta Ohm HD 2102.2) with a probe in the spectral range $280-315 \mathrm{~nm}$ and the dose levels was tuned changing the pulse duration in the range 350 min. Lock-in thermography was used to evaluate the effect of the UV-B radiation on the physiological state of the plant. The leaves were heated by the use of an halogen lamp $(200 \mathrm{~W})$ square wave modulated with a period $\mathrm{T}=40 \mathrm{~s}(25$ $10^{-3} \mathrm{~Hz}$ ). During heating, thermal images were acquired with a frame rate of $5 \mathrm{~Hz}$. The analysis was realized before and after UV-B irradiation and phase images were calculated in both case. 


\section{Results and discussion}

Figure 1-A shows a sequence of thermal images of a leaf recorded during and after UV-B irradiation (dose $3.6 \mathrm{~kJ} / \mathrm{m}^{2}$ ). It represents an example of the typical thermal behavior observed on all leaves irradiated. The images clearly point out as after irradiation the temperature of the leaf decrease up to a temperature lower respect to the start value. A maximum decrease of about $-2.3^{\circ} \mathrm{C}$ can be estimate from the temporal trend of the average temperature variation in figure 1-B calculated on the whole leaf. This particular cooling effect was observed on all the leaves irradiated with doses starting from $1.0 \mathrm{~kJ} / \mathrm{m}^{2}$. As visible in the same trend, the cooling start after about some minutes (depending on the applied dose) of irradiation and its effects become more and more evident when the UV-B stimulation ends. This peculiar thermal response of the plant to the UV-B radiation could be due to a defense mechanism that allow to increase the amount of sap, and in particular of water, present in the irradiated areas.

A

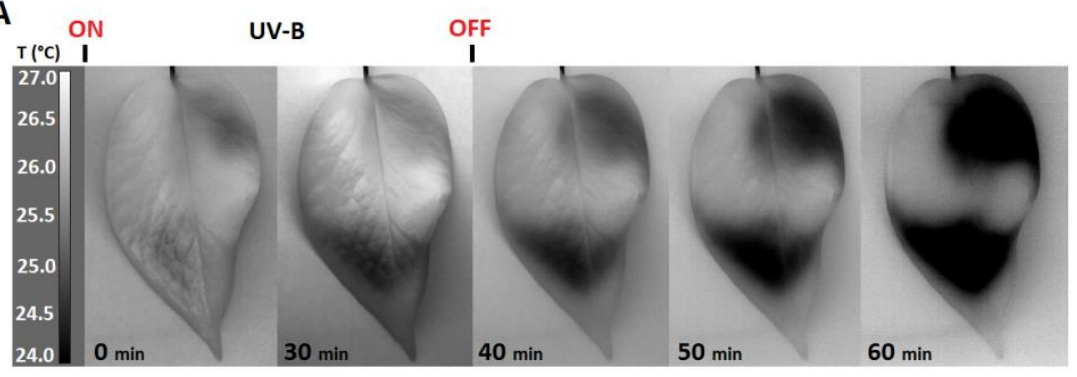

B

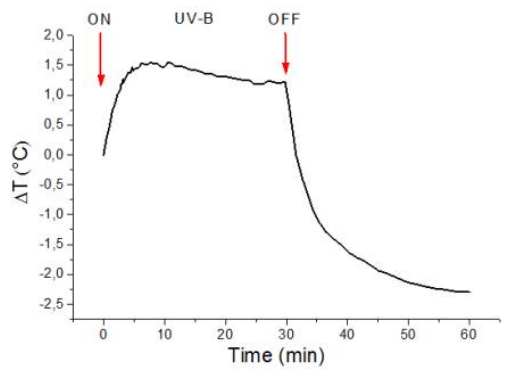

Fig. 1: Thermal behaviour of a leaf under UV-B irradiation (30 min, total dose $\left.3.6 \mathrm{~kJ} / \mathrm{m}^{2}\right)$. A) Sequence of thermal images of the leaf during and after irradiation. B) Temporal trend of the mean temperature variation evaluated on the whole leaf.

This explanation is supported by the results achieved with the lock-in thermography. In this analysis, for thermal stimulation with period T much longer than the time constant $r$ of the leaf, the phase $\rho(\omega)$ can be approximated by $\rho(\omega) \approx \omega \tau$ [6]. In particular, $T$ can be considered proportional to the heat capacity and so, to the leaf water content. Figure 2 shows the comparison of the phase images achieved on some leaves before and after UV-B irradiation with different doses. As the images show, in all cases, after the irradiation, the phase and so the water content, increase in different regions of the leaves and the difference of water between before and after appears to be greater for larger doses (figures 2-A, 2-B, 2-C). Furthermore, it was observed that for doses up to $3.6 \mathrm{~kJ} / \mathrm{m}^{2}$ the thermal process appears reversible and the leaves gradually return to their initial state while for the higher dose of $6.0 \mathrm{~kJ} / \mathrm{m}^{2}$ (figure $2-\mathrm{D}$ ) permanent damages was observed and the process became irreversible.

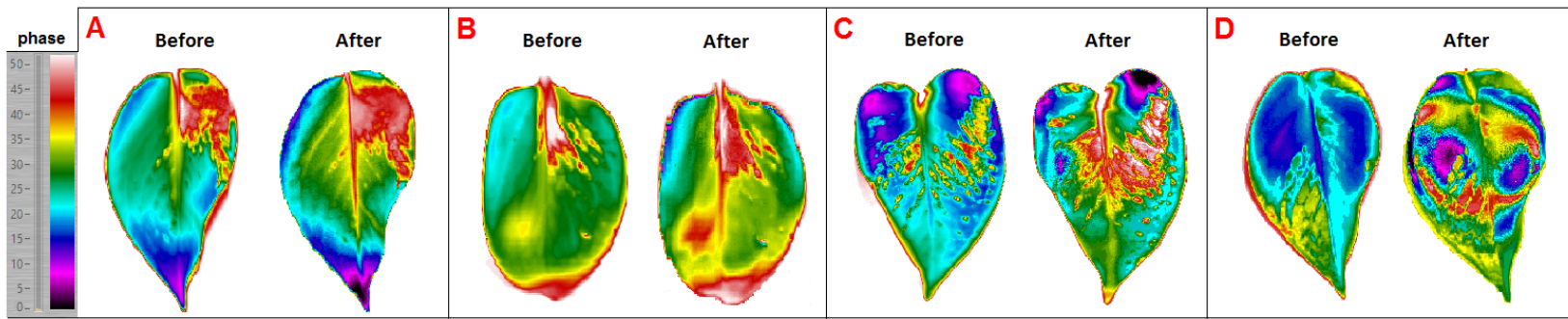

Fig. 2: Phase images achieved before and after different UV-B doses $\left(\mathrm{kJ} / \mathrm{m}^{2}\right):$ A) 0.4, B) 1.0, C) 3.6, D) 6.0.

\section{Conclusion}

The UV-B - plants interaction analyzed by the use of active thermography allows to highlight new aspects of the response mechanisms in presence of this radiation. In particular, a colling effect of the leaves was observed as defense to the UV-B irradiation. Our achievements emphasize the importance that thermography can have for real-time analysis of the plants response under external stimulation and they open new opportunites for the use of this tecnique in the vegetable world. 


\section{REFERENCES}

[1] Schreiner M, Mewis I, Huyskens-Keil S, et al. UV-B induces Secondary Plants Metabolites - Potential Benefits for Plants and Human Health. Critical Reviews in Plant Sciences 2012, 31:3, 229-240.

[2] Hollosy F. Effects of ultraviolet radiation on plant cells. Micron 33 (2002), 179-197.

[3] Shama S, Chatterjee S, Kataria S, et al. A Review of Plants to UV-B Radiation Related Stress. UV-B Radiation: From Environmental Stressor to Regulator of Plant Growth, book, ch.5, April 2017, Wiley-Blackwell.

[4] Robson TM, Klem K, Urban O, Jansen MAK. Re-interpreting plant morphological responses to UV-B radiation. Plant, Cell and Environment (2015) 38, 856-866.

[5] Ishimwe R, Abutaleb K, Ahmed F. Applications of Thermal Imaging in Agriculture-A Review. Advances in Remote Sensing, 2014, 3, 128-140.

[6] Garbea CS, Schurrb U, Jähnea B. Thermographic measurements on plant leaves. Proceedings Volume 4710, Thermosense XXIV; (2002); doi: 10.1117/12.459590. 